\title{
Ciclo de melhoria de qualidade para aumentar a obtenção de consentimento informado em procedimentos de planeamento familiar
}

Matilde Padrão Dias, ${ }^{1}$ Cecília Shinn, ${ }^{2}$ Liliana Amaral, ${ }^{3}$ Maria João Araújo, ${ }^{4}$ Ana Pinto, ${ }^{5}$ Paula Gonzalez, ${ }^{6}$ Daniel Pinto ${ }^{7}$

\begin{abstract}
RESUMO
Objetivos: A equipa da Unidade de Saúde Familiar (USF) S. Julião verificou que o consentimento informado não era obtido nos procedimentos de planeamento familiar - colocação de dispositivo intra-uterino e de implante subcutâneo. Foi criada uma equipa de investigação para realizar um ciclo de melhoria de qualidade, com o objetivo de aumentar a taxa de obtenção de consentimento informado. Tipo de estudo: Estudo pré-experimental, pré- e pós-intervenção, sem grupo controlo.

Local: USF S. Julião.

População: Utentes da USF S. Julião que coloquem dispositivo intra-uterino ou implante contracetivo.

Métodos: Em agosto de 2012 já tinha sido adotado um formulário de consentimento e nos meses seguintes foi obtido consentimento em $62,5 \%$ dos procedimentos, o que foi considerado insuficiente. A equipa de investigação organizou a realização de grupos focais em março de 2013 para definir estratégias de melhoria. O outcome primário foi a taxa de obtenção de consentimento em procedimentos de planeamento familiar. Foi estabelecida a meta de $95 \%$ e a adesão às estratégias foi monitorizada. Calculou-se uma dimensão amostral pós-intervenção de 18 utentes. Definiram-se dois períodos pós-intervenção: imediato ( 0 a 3 meses) e tardio (4 a 6 meses). Utilizou-se o teste exato de Fisher para comparar a taxa de obtenção do $\mathrm{Cl}$ com o período pré-intervenção.

Resultados: A monitorização das estratégias definidas revelou o cumprimento total das mesmas. Foi obtido o consentimento em todos os procedimentos de planeamento familiar realizados tanto no período pós-intervenção imediato como no pós-intervenção tardio, em comparação com $62,5 \%$ no período pré-intervenção $(p<0,001)$.

Conclusões: Os profissionais da USF conseguiram implementar as estratégias e melhorar a obtenção de consentimento. O envolvimento de toda a equipa poderá ter aumentado a eficácia da intervenção. A aplicação das estratégias em apenas uma unidade limita a generalização dos resultados. Contudo, estratégias simples parecem aumentar significativamente a taxa de obtenção do consentimento.
\end{abstract}

Palavras-chave: Consentimento Informado; Planeamento Familiar; Melhoria da Qualidade.

\section{INTRODUÇÃO}

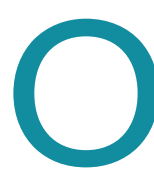
s princípios éticos - beneficência, não maleficência, autonomia e justiça - que guiam a prática médica baseiam-se no respeito pela vida e pela pessoa. A proteção do ser humano, da sua dignidade e identidade implica a garantia de que toda a pessoa, sem discriminação, vê respeitados os seus direitos e liberdades fundamentais. ${ }^{1} \mathrm{O}$ consentimento informado é um dos pontos de partida para uma prática médica baseada no respeito pela autonomia e autodeterminação da pessoa. É objetivo do consentimento informado promover a participação e autorização esclarecida, ativa e autónoma da pessoa no processo de decisão clínica.

O conceito de consentimento usado em saúde encontra uma correlação com o conceito jurídico, em que consentir significa declarar, sem qualquer tipo de interferência na constituição da vontade e após compreensão da in-

1Médica interna de Medicina Geral e Familiar, USF Dafundo, ACES Oeiras ${ }^{2}$ Médica interna de Medicina Geral e Familiar, USF S. Julião, ACES Oeiras ${ }^{3}$ Médica interna de Medicina Geral e Familiar, USF S. Julião, ACES Oeiras ${ }^{4}$ Médica interna de Medicina Geral e Familiar, USF Conde de Oeiras, ACES Oeiras ${ }^{5}$ Secretária clínica, USF S. Julião, ACES Oeiras

${ }^{6}$ Enfermeira, USF S. Julião, ACES Oeiras

${ }^{7}$ Médico especialista de Medicina Geral e Familiar, USF S. Julião, Departamento de Medicina Geral e Familiar - Faculdade de Ciências Médicas - Universidade Nova de Lisboa 
formação fornecida pelo profissional de saúde. ${ }^{2}$ Assim, não é suficiente apenas transmitir a informação, mas também deverá garantir-se a compreensão da mesma.

Em Portugal, ao abrigo do "princípio da liberdade declarativa", a decisão individual de aceitação ou recusa de determinada intervenção em saúde deve ser expressa verbalmente, salvo as exceções previstas na lei. ${ }^{2}$ Está ainda prevista a forma escrita de expressão do consentimento em procedimentos regulamentados pela Direção-Geral da Saúde (DGS), nomeadamente na colocação de dispositivos anticoncetivos. ${ }^{3}$

O consentimento informado, obtido formal e sistematicamente, como parte das boas práticas de uma instituição atual, garante respeito pela autonomia e dignidade das utentes e proteção legal para os profissionais de saúde.

Nos últimos anos tem-se verificado uma maior consciencialização da importância da avaliação e monitorização dos cuidados prestados. Neste sentido têm sido desenvolvidas diversas ferramentas, como a grelha Diagnóstico do Desenvolvimento Organizacional nas USF (DiOrUSF), que permite, através da avaliação e monitorização contínuas, melhorar o desempenho da unidade, a satisfação dos cidadãos e dos profissionais de saúde. No item relativo à "comunicação com os utentes", o critério $Q$ descreve os procedimentos para os quais o registo do consentimento é mandatório, a saber: colocação de DIU e implantes, iso-imunização Rh, administração da VASPR, autoscopia, pequenas cirurgias, participação em exames finais de internato de especialidade, recolhas de imagem e participação em investigação. ${ }^{4}$

No seguimento da aplicação da grelha DiOr-USF na Unidade de Saúde Familiar (USF) S. Julião - Agrupamento de Centros de Saúde (ACES) Lisboa Ocidental e Oeiras constatou-se que a obtenção do consentimento informado para procedimentos de planeamento familiar (PF) colocação de dispositivo intrauterino (DIU) ou de implante subcutâneo - estaria a ser deficitária, tendo os profissionais de saúde admitido que não tinham a prática habitual de obter o consentimento. Constatou-se ainda que não existia um registo sistemático de registo que permitisse a sua monitorização e avaliação.

A situação foi discutida em reunião de equipa durante o mês de agosto de 2012. Foi criado um documento específico de consentimento informado para cada procedimento (baseado no documento elaborado pela Comissão de Ética da Administração Regional de Saúde do Norte) ${ }^{5}$ e decidiu-se alterar a base de dados de registo de colocação de DIU e implante criada pela equipa da USF em 2008, acrescentando-se uma coluna para registo (com a opção de "sim" ou "não") da obtenção do respetivo consentimento.

Reconhecendo a pertinência do tema, a equipa decidiu, em março, avaliar se o problema se mantinha. Verificou-se que houve um aumento progressivo da taxa de obtenção do consentimento nos primeiros três meses após a reunião de agosto, tendo a taxa estabilizado nos meses seguintes (71,4\% em março).

Estes resultados foram apresentados na reunião mensal da USF no final de março de 2013 e, apesar do aumento da obtenção do consentimento, constatou-se que criar um registo da obtenção do consentimento não tinha sido suficiente para atingir um patamar considerado satisfatório. Assim, foi tomada a decisão de criar alternativas para complementar a estratégia inicial.

Foi criada uma equipa de investigação (os autores) responsável por planear e executar um ciclo de melhoria de qualidade, com o objetivo principal de aumentar a taxa de obtenção de consentimento informado em procedimentos de PF. Assim, este trabalho pretende avaliar a eficácia das estratégias definidas pela equipa da USF no aumento da taxa de obtenção do consentimento informado.

\section{MÉTODOS}

As USF são pequenas unidades operativas dos Centros de Saúde com autonomia funcional e técnica, que contratualizam objetivos de acessibilidade, adequação, efetividade, eficiência e qualidade e que garantem aos cidadãos inscritos uma carteira básica de serviços. ${ }^{6}$

A USF S. Julião localiza-se num meio urbano e é constituída por uma equipa de dez médicos de família, oito enfermeiros e seis secretárias clínicas. Segundo o relatório de avaliação da contratualização em cuidados de saúde primários da Administração Regional de Saúde de Lisboa e Vale do Tejo (Análise Retrospetiva de 2007/2011), constitui uma das 14 USF, em 85, que cumpriu na totalidade os indicadores contratualizados. ${ }^{7}$

Nesta USF, os procedimentos de PF são realizados em conjunto pelo médico e enfermeiro de família. A utente é informada pelo médico e/ou enfermeiro sobre as diversas opções disponíveis e toma uma decisão esclarecida sobre o método a utilizar. O consentimento é entregue e lido, são esclarecidas as dúvidas e é preenchido em duplicado antes da realização do procedimento. Após a realização do procedimento, o médico ou enfermeiro faz o registo na base de dados da USF e arquiva o consentimento no processo da utente. 
Após reconhecimento, por parte da equipa da USF, da necessidade de melhoria na obtenção dos consentimentos, a equipa de investigação encorajou a criação de grupos focais com todos os profissionais durante uma das suas reuniões. Estes grupos foram facilitados por dois dos investigadores e tinham como objetivo discutir possíveis estratégias para melhorar a obtenção do consentimento informado. Os profissionais presentes na reunião foram divididos em dois grupos e foram discutidas possíveis estratégias durante 20 minutos. Cada grupo apresentou as suas conclusões, tendo-se chegado a um consenso sobre as medidas a executar.

De entre os membros da equipa da USF definiram-se os responsáveis por implementar e garantir a continuidade de cada medida. Foi também pedido à equipa para definir a meta a alcançar na obtenção do consentimento, tendo sido acordada uma taxa de $95 \%$.

Para avaliar a eficácia da intervenção foi desenhado um estudo pré-experimental, tipo pré- e pós-intervenção, sem grupo controlo, sendo o outcome primário do estudo a taxa de obtenção de consentimento informado nos procedimentos de PF no período imediato após a intervenção. O outcome secundário foi definido como a taxa de obtenção de consentimento nos procedimentos no período tardio após a intervenção. Este outcome permite avaliar se os efeitos da intervenção são mantidos ao longo do tempo. A população a estudar consistiu nas utentes da USF S. Julião do sexo feminino que colocaram DIU/implante.

A avaliação do outcome primário do estudo foi realizada através da análise do registo da obtenção do consentimento pelos profissionais da USF. Se o registo fosse "sim" considerava-se que o consentimento foi preenchido, se o registo fosse "não" ou estivesse em branco considerava-se que o consentimento não tinha sido obtido.

O estudo comparou a obtenção do consentimento antes e depois da implementação das estratégias definidas pela equipa da USF. Na hipótese nula não se verifica diferença na taxa de obtenção antes e depois da intervenção; na hipótese alternativa, a taxa de obtenção dos consentimentos é superior após a intervenção realizada.

$\mathrm{O}$ período estudado foi dividido em quatro fases. A fase de diagnóstico do problema compreende o período entre agosto (momento em que foi identificado o problema) e outubro de 2012 (quando passaram a ser obtidos os consentimentos para parte significativa dos procedimentos). A fase pré-intervenção compreende o período entre novembro de 2012 e março de 2013, em que se verificou uma estabilização na taxa de obtenção do consentimento. A fase pós-intervenção imediata compreende o período entre abril (início da intervenção) e junho de 2013 (obtenção da amostra para o outcome primário). A fase pós-intervenção tardia compreende o período entre julho e setembro de 2013 (avaliação do outcome secundário).

Foi feita uma comparação com o controlo histórico através do teste exato de Fisher.

A taxa de obtenção de consentimento informado na fase pré-intervenção foi de 62,5\% entre novembro de 2012 e março de 2013 (após a estabilização dos valores das taxas de obtenção do consentimento). A dimensão da amostra foi calculada para poder demonstrar um aumento de 62,5 para $95 \%$ na taxa de obtenção do consentimento, assumindo um erro do tipo alfa de $5 \%$ e uma potência de $90 \%$ e que não haveria dados em falta. Tendo em conta que já tinham sido realizados 40 procedimentos pré-intervenção, seriam necessárias 18 utentes na fase pós-intervenção. Escolheu-se uma amostra mínima de 20 utentes, uma vez que este é o número inteiro mínimo que permite atingir uma taxa de cumprimento entre $95 \%$ e $100 \%$. Na fase pré-intervenção verificou-se uma média de 8 procedimentos por mês, pelo que cada fase pós-intervenção teve uma duração prevista de 3 meses (24 procedimentos esperados).

Para monitorizar a adesão às estratégias de melhoria delineadas, os elementos da equipa de investigação registaram mensalmente o cumprimento das mesmas. Este registo foi realizado nos dois períodos pós-intervenção referidos. Os elementos que procederam à recolha destes dados limitaram-se à observação do cumprimento das estratégias, não tendo qualquer tipo de interferência no comportamento dos profissionais.

Para garantir a confidencialidade e anonimato das utentes e dos profissionais de saúde, a colheita dos dados relativos à obtenção do consentimento foi realizada pela coordenadora da USF S. Julião no final do período em estudo, sendo fornecido à equipa de investigação um ficheiro digital onde constava apenas a data, o tipo de procedimento realizado e os dados incluídos na coluna para registo do consentimento. Assegurou-se, assim, que os investigadores apenas tiveram acesso a dados anonimizados. Os profissionais de saúde da USF S. Julião deram consentimento para participação no estudo.

O protocolo do estudo foi aprovado pela Diretora Executiva do ACES Lisboa Ocidental, pela Coordenadora da USF S. Julião e pela Comissão de Ética da Administração Regional de Saúde de Lisboa e Vale do Tejo. O estudo não implicou qualquer custo para o ACES, não interferiu com 
o funcionamento regular da unidade, nem prejudicou a qualidade dos serviços prestados.

\section{RESULTADOS}

Participaram nos grupos focais 15 dos 24 profissionais da USF, tendo sido definidas as seguintes estratégias a implementar:

1. Colocação dos DIU e implantes dentro de uma caixa colorida na sala de PF;

2. Criação de dossier com consentimentos por preencher, colocado em cima da caixa colorida;

3. Colocação de novas cópias de consentimentos por preencher sempre que se retira a última;

4. Colocação de recordatório na caixa colorida (Figura $1)$.

A monitorização das estratégias revelou o total cumprimento das mesmas (Quadro I).

Na fase pós-intervenção imediata foram realizados 26 procedimentos e na fase pós-intervenção tardia 25 proce-

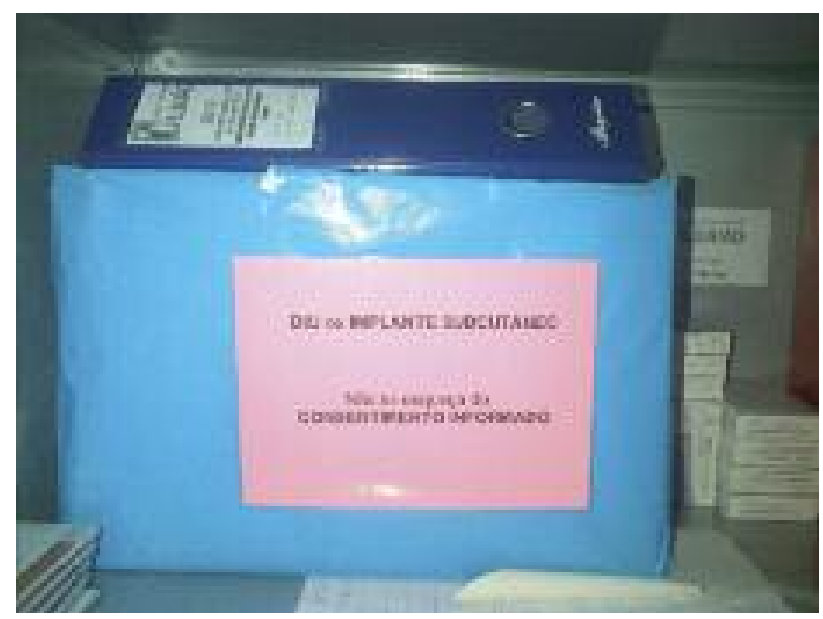

Figura 1. Estratégias definidas pela equipa da USF S. Julião. dimentos. Foi obtido o consentimento informado na totalidade dos procedimentos em ambas as fases, comparativamente a $62,5 \%$ na fase pré-intervenção (Figura 2). A taxa de obtenção do consentimento informado dos períodos pós-intervenção imediato e tardio foi comparada com o período pré-intervenção através do teste exato de Fisher, tendo sido encontrada uma diferença estatisticamente significativa em ambos os períodos $(p<0,001)$.

\section{DISCUSSÃO}

O reconhecimento do problema por parte de todos os profissionais da USF permitiu o envolvimento de todos os seus elementos e o trabalho em equipa proporcionou o sucesso atingido. A escolha de medidas simples e fáceis de executar e manter resultou numa melhoria da obtenção do consentimento informado nos procedimentos de PF, melhorando a participação das utentes de forma esclarecida, ativa e autónoma no processo de decisão clínica. $\mathrm{O}$ aumento da taxa de obtenção do consentimento permitiu melhorar o cumprimento dos itens da grelha DiOr-USF.

Este estudo apresenta, como principais pontos fortes, a fácil implementação das medidas selecionadas, o reforço de cuidados de saúde que respeitem a autonomia das utentes e a consequente melhoria da prestação de cuidados (reforço da relação médico-doente, investimento no processo comunicacional, contínuo e participado e importância da decisão livre e esclarecida).

As estratégias adotadas são fáceis de aplicar a uma equipa madura, mas pela sua simplicidade seriam igualmente fáceis de aplicar a uma equipa menos experiente, desde que motivada para a mudança. A metodologia escolhida para melhoria da taxa de obtenção do consentimento informado pode ser aplicada a outros procedimentos que carecem de consentimento e estão relacionados com a utilização de material clínico específico, como procedimentos de pequena cirurgia e administração de Imunoglobulina anti-D.

As medidas implementadas demonstraram ser de elevada eficiência, uma vez que não implicaram custos materiais e de tempo significativos.

Foi realizada uma pesquisa na PubMed com os descritores MeSH "Informed Consent" e "Quality Improvement", não tendo sido encontrados estudos semelhantes a este trabalho. Os estudos encontrados abordam principalmente a qualidade da informação prestada 


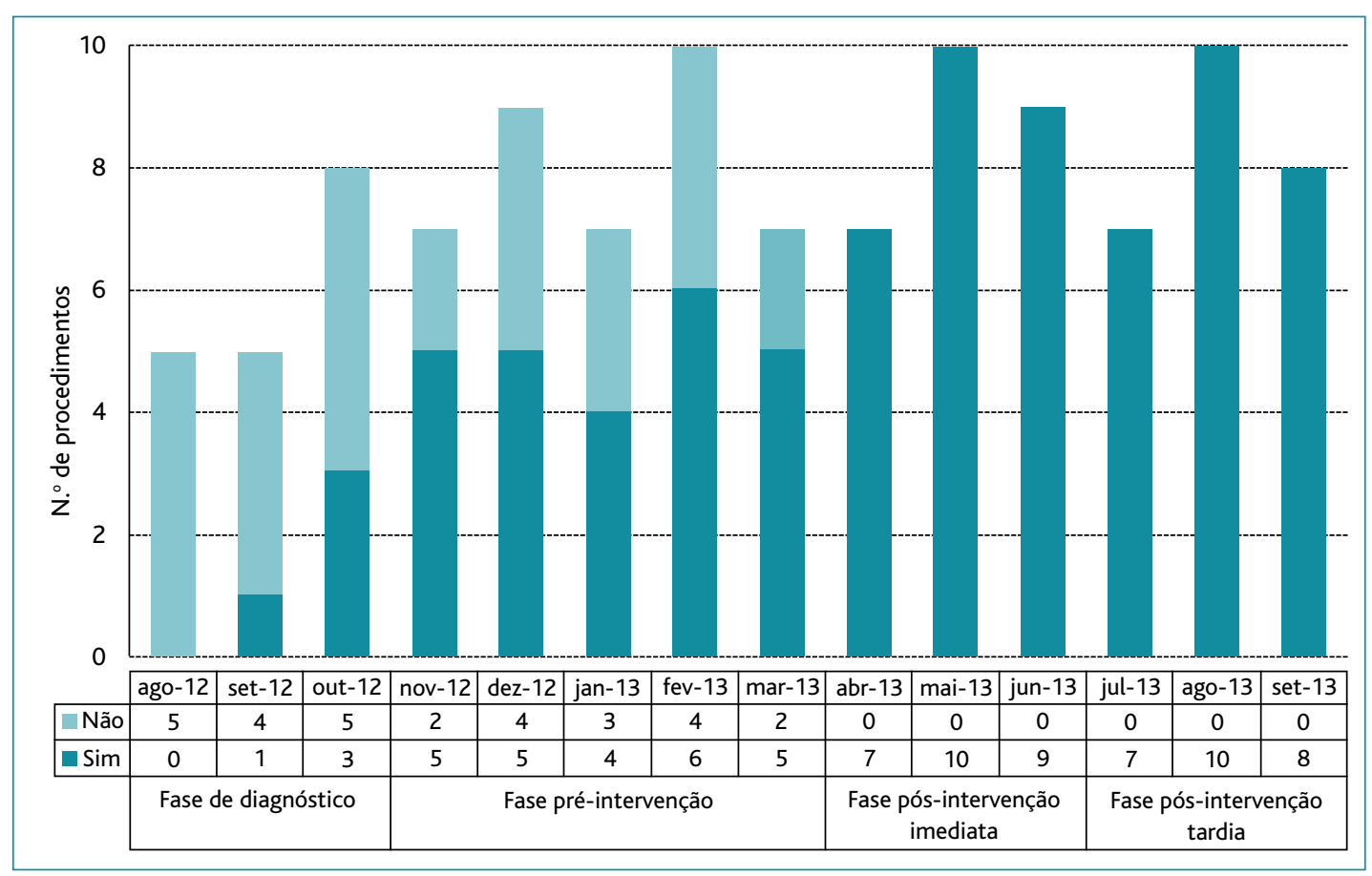

Figura 2. Total de procedimentos de PF realizados e respectivos consentimentos informados obtidos.

através do consentimento e o seu impacto na qualidade dos cuidados médicos e decisões dos utentes. ${ }^{8-9}$

Tal como já referido, não se revelou ser necessário obter consentimento das utentes para participação no estudo uma vez que foi adotada como estratégia o fornecimento dos dados através da coordenadora, a fim de garantir o anonimato dos mesmos.

Uma limitação importante deste estudo relaciona-se com o facto de ser baseado em registos. Na prática, a realização de um procedimento de PF pode não corresponder ao preenchimento da base de dados. No entanto, este registo já era realizado pelos profissionais desta unidade aquando da colocação de DIU e implantes. Além disso, na discussão nos grupos focais os profissionais referiram ser pouco provável existirem falhas no preenchimento deste registo. Assim, a equipa de investigação considerou que não existiriam procedimentos não registados nem procedimentos registados que não tenham sido realizados.

Os investigadores optaram por não consultar os processos clínicos individuais para verificar a fiabilidade dos registos, uma vez que não foi pedida autorização às utentes em causa.

As estratégias utilizadas foram adaptadas à realidade organizacional da USF S. Julião, o que pode limitar a repro- dutibilidade do estudo com os mesmos níveis de eficácia em unidades com diferentes modelos de organização (por exemplo, em que haja um único profissional a realizar estes procedimentos ou em que haja mais de uma sala de $\mathrm{PF})$.

Apesar dos profissionais terem continuado a obter o consentimento informado em todos os procedimentos realizados nos seis meses seguintes à intervenção, é possível que o seu efeito seja reduzido a longo prazo. Após a realização do estudo, a monitorização dos resultados pode ser conseguida parcialmente através do preenchimento da grelha DiOr-USF. No entanto, a manutenção da monitorização das estratégias definidas não foi prevista inicialmente pelos investigadores, pelo que se propôs posteriormente a sua alocação a um elemento da USF.

Uma intervenção simples, partindo de soluções encontradas pelos profissionais da unidade, levou a melhorias significativas na taxa de obtenção do consentimento informado para procedimentos de PF. A intervenção descrita poderá ser replicada noutras unidades de saúde com baixas taxas de obtenção de consentimento informado. Para os resultados encontrados contribuiu o empenho de toda a equipa, a distribuição inequívoca das tarefas a executar e a simplicidade das medidas implementadas. 


\section{REFERÊNCIAS BIBLIOGRÁFICAS}

1. World Medical Association. World Medical Association Declaration of Helsinki: ethical principles for medical research involving human subjects. JAMA 2013 [cited 2014 Jan 22];310(20):2191-4. Available from: http://jama.jamanetwork.com/article.aspx?articleid=1760318

2. Comissão de Ética para a Saúde. CES-ARSLVT - Ética assistencial: parecer n 009/2012. Lisboa: ARSLVT; 2012 [cited 2013 Jul 26]. Available from: http://www.arslvt.min-saude.pt/ces/Documents/Parecer_ConsInformadoAprovado_2013-02-15AFV\%20(2).pdf

3. Comissão de Ética para a Saúde. CES-ARSLVT - Ética Assistencial: parecer sobre pedido de informação relativo a situações relacionadas com a prática clínica que não dispensam o consentimento informado (parecer $n^{\circ}$ 009/2012). Lisboa: ARSLVT; 2012 [cited 2013 Jul 26]. Available from: http://www.arslvt.min-saude.pt/ces/Documents/Anexo\%20I\%20-\%20revisto-VFF\%20(2).pdf

4. Administração Central do Sistema de Saúde. Guia para aplicação do diagnóstico do desenvolvimento organizacional nas USF: DiOr-USF. Lisboa: Ministério da Saúde; 2012 [cited 2013 Jul 26]. Available from: http://www.acss.min-saude.pt/Portals/0/Guia\%20DiOr-USF.pdf

5. Administração Regional de Saúde do Norte. Modelos de consentimento informado, livre e esclarecido. Porto: ARSN; 2009 [cited 2013 Jul 29]. Available from: http://portal.arsnorte.minsaude.pt/portal/page/portal/ARSNorte/Comiss\%C3\%A30\%20de\%20\%C3\%89tica/Ficheiros/Consentimento\%20Informado/7A4D4ADDB2579BA4E040140A110272B3

6. Unidades de Saúde Familiar - Associação Nacional. Unidades de saúde familiar. Porto: USFAN [cited 2013 Jul 29]. Available from: http://www.usfan.pt/index.php/unidades
7. Departamento de Planeamento e Contratualização. Relatório de avaliação da contratualização em cuidados de saúde primários - Unidades de saúde familiar (USF) e unidades de cuidados de saúde personalizados (CSP), ano 2011: análise retrospetiva dos resultados de 2007/2011. Lisboa: Administração Regional de Saúde de Lisboa e Vale do Tejo; 2012 [cited 2013 Jul 29]. Available from: http://www.arslvt.min-saude.pt/ARSLVT/EstruturaOrganica/Documents/RELAT\%C3\%93RIO\%20AVALIA\%C3\%87\%C3\%830\%202 011\%20USF\%20E\%20UCSP.pdf

8. Force RW, Keppel GA, Guirguis-Blake J, Gould DA, Vincent C, Chunchu K, et al. Contraceptive methods and informed consent among women receiving medications with potential for adverse fetal effects: a Washington, Wyoming, Alaska, Montana, Idaho (WWAMI) region study. J Am Board Fam Med. 2012;25(5):661-8.

9. Doyle JL, Kenny TH, von Gruenigen VE, Butz AM, Burkett AM. Implementing an induction scheduling procedure and consent form to improve quality of care. J Obstet Gynecol Neonatal Nurs. 2012;41(4):462-73.

\section{CONFLITOS DE INTERESSES}

Os autores declaram não ter conflitos de interesses.

\section{ENDEREÇO PARA CORRESPONDÊNCIA}

Matilde Padrão Dias

Rua Quinta da Formiga, n¹3 3ºdto 1495-170 Algés

E-mail: matildepadraodias@gmail.com

Recebido em 06-02-2014

Aceite para publicação em 17-06-2014

Versão corrigida a 05 /09/2014 (inclui duas figuras omissas na versão original)

\section{ABSTRACT}

\section{INFORMED CONSENT FOR FAMILY PLANNING - A QUALITY IMPROVEMENT STUDY}

Objectives: To increase the rate of obtaining informed consent for family planning procedures (insertion of intrauterine devices and subcutaneous contraceptive implants)

Study design: Uncontrolled pre- and post- intervention design

Setting: S. Julião Family Health Unit.

Participants: Patients in the S. Julião FHU who visited the Unit for insertion of an intrauterine device or subcutaneous contraceptive implant.

Methods: The S. Julião FHU team designed an informed consent form in August 2012 but usage rates were found to be low (62.5\%). Focus groups were organized to propose quality improvement strategies to increase the rate of obtaining informed consent. Two post-intervention periods were defined: immediate ( 0 to 3 months) and late ( 4 to 6 months). The Fisher exact test was used to compare rates of informed consent obtained before and after the intervention.

Results: Informed consent was obtained from $62,5 \%$ of patients in the pre-intervention period. Informed consent was obtained for $100 \%$ of family planning procedures during both the immediate and late post intervention periods $(p<0.001)$.

Conclusions: Health professionals at S. Julião FHU were able to implement quality improvement strategies and to increase rates of obtaining informed consent. The efficacy of the intervention was due to involvement of all staff members. This strategy was only applied in one Family Health Unit, which may limit the external validity of the results. However, simple strategies may be effective in significantly increasing rates of obtaining informed consent in family planning procedures.

Keywords: Informed Consent; Family Planning; Quality Improvement. 